\title{
Effect of Dietary Corn Oil, Butter and Beef Tallow on the Cecal Microflora and Short-Chain Fatty Acids in Rats
}

\author{
Yoshiyuki Morishita and Kimiko SHIROMIZU \\ Department of Biomedical Food Research, National Institute of Health, \\ Toyama 1-23-1, Shinjuku-ku, Tokyo, 162 Japan
}

(Received for publication, November 8, 1995)

\begin{abstract}
Wistar rats fed apurified diet containing 5\% corn oil, butter, or beef tallow for 4 weeks were examined for cecal microflora and cecal short-chain fatty acids. Bifidobacteria and proteus showed remarkable decreases in viable numbers in the cecum of rats fed a butter-containing diet compared with animals fed corn oil- or beef tallow-containing diets. Clostridia showed a significantly decreased number in the beef tallow and butter groups compared with the corn oil group. The concentrations of acetate, propionate, and butyrate and the total concentrations of short-chain fatty acids in the cecum of rats were significantly lower in the rats given butter or beef tallow compared with the animals given corn oil. The cecal $\mathrm{pH}$ was higher in the butter and beef tallow groups. The results indicate that butter and beef tallow as dietary fat are unfavorable for bacterial fermentation in the cecum of rats.
\end{abstract}

Key words: dietary fats; cecal microflora; short-chain fatty acids; rats

Dietary fat is one of the main nutrients to produce large energy and essential fatty acids for human and animals, and the type and amount of dietary fat have different effects on them. In particular, the epidemiological data and animal experiments show that there is a significant relationship between the type and amount of dietary fat and mammary and colonic carcinogenesis (1, 12). On the other hand, fat and fatty acids are known to have antibacterial activities depending on structures and deterioration $(2,3,5,10,11$, 13, 14). Therefore, the different types of dietary fat in normal dose might have different effects on the intestinal microflora and microbial metabolism, even at normal concentrations, although high fat diets were reported to have some effects on the bacterial composition of the fecal microflora in the pig (7) and the isolation rate of Clostridium perfringens in the feces of rats (4).

We are investigating the effect of dietary fats and oils on the microflora and on the production of short-chain fatty acids in the large intestine of experimental animals since they differ in the proportion of component fatty acids. The present study demonstrated the cecal microflora and short-chain fatty acid concentrations in rats fed a purified diet containing corn oil, butter and beef tallow among the different fats and oils.

Three-week old female Wistar rats (Shizuoka Laboratory Animal Center, Shizuoka) were used and maintained as described previously (9). Two rats were housed in a metal cage with a stainless steel mesh bottom, and allowed free access to test diets and water for 4 weeks after 4 days of acclimation on the basal diet containing $5 \%$ corn oil. Six rats were used for each diet group. The purified diet used was composed of $23 \%$ casein, $67 \%$ corn starch, $5 \%$ fat, $1 \%$ vitamin mixture, $4 \%$ mineral mixture, and $5 \%$ cellulose. The dietary fats used were corn oil as basal fat, butter without salt, 


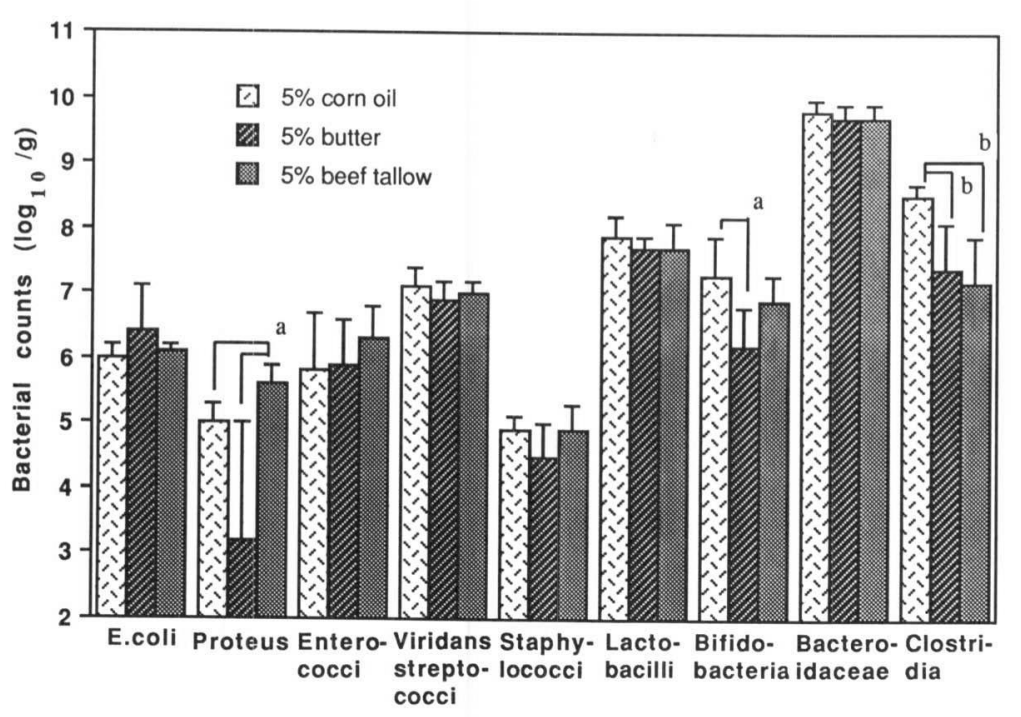

Fig. 1. Effect of dietary fat on the cecal microflora of rats. ${ }^{\mathrm{a}, \mathrm{b}}$ Significant difference at $p=0.05$ and 0.01 , respectively.

and beef tallow. The diets were prepared in pellets and vacuum-sealed in plastic bags as described by Morishita et al (9).

After 4 weeks of feeding, the cecal contents were aseptically collected from rats sacrificed with $\mathrm{CO}_{2}$. One part of the sample was used for bacteriological examination and other half was used to measure $\mathrm{pH}$ and short-chain fatty acid (SCFA) concentrations. For bacteriological analysis, the samples were diluted in 10-fold steps with phosphate buffer $(\mathrm{pH} 7.2)$ containing $0.05 \%$ cysteine $\mathrm{HCl}$ (6) and 0.05-ml volumes of appropriate dilutions were spread on the surface of agar plates of three non-selective and seven selective media, which were prepared as described by Mitsuoka et al (6) and Morishita and Miyaki $(8)$. Anaerobic cultivation was performed at $37^{\circ} \mathrm{C}$ in steel jars with an atmosphere of $10 \% \mathrm{CO}_{2}$ and $90 \% \mathrm{~N}_{2}(9)$. Colony counts were performed and bacteria were broadly classified as described in the previous report $(8)$. For $\mathrm{pH}$ determination, the cecal samples were measured with a $\mathrm{pH}$ meter (Toa Electronics, Tokyo) after diluting 1:3 in Milli-Q water (Japan Millipore Ltd, Tokyo) immediately after sampling. After that, the cecal suspensions were stored at $-20^{\circ} \mathrm{C}$. For measurement of SCFAs, the cecal suspensions were thawed and centrifuged at 10,000 rpm for $12 \mathrm{~min}$, and the supernatants were analysed by gas-liquid chromatography as described previously (9). All data are expressed as means with standard deviations and were analysed by Student's $t$-test.

As shown in Fig. 1, bifidobacteria showed larger numbers in the cecum of rats fed a corn oil diet than the butter and beef tallow diets; the number of bifidobacteria in rats fed the butter diet $(6.2$ in average logarithmic count/g) significantly decreased compared to animals fed the corn oil diet (7.2/g, $p<0.05)$, and also moderately, but not significantly, decreased compared to animals fed the beef tallow diet $(6.9 / \mathrm{g}, p=0.059)$. Proteus showed a significantly larger number $(5.6 / \mathrm{g})$ in the beef tallow group than in the butter and corn oil groups $(p<0.05)$ while the organisms were found in decreased numbers $(3.2 / \mathrm{g})$ in the butter group $(p=$ $0.057)$ compared to the corn oil group. The number of cecal clostridia, not including $C$. perfringens, was significantly larger in rats given corn oil $(p<$ $0.001)$ than rats given the other fats. There were no significant differences in the number of cecal Escherichia coli between the three groups, but $E$. coli was found in larger numbers $(6.6$ and $7.4 / \mathrm{g})$ in two of the five animals fed a butter diet than in the other animals examined (less than 6.5 in $\log _{10}$ counts/g). The other bacterial groups, such as viridans streptococci, staphylococci, lactobacilli, and bacteroidaceae, showed no significant differences in viable number between the experimental 
Table 1. Effect of dietary fat on the concentrations of cecal short-chain fatty acids in rats

\begin{tabular}{lccc}
\hline & $5 \%$ corn oil & $5 \%$ butter & $5 \%$ beef tallow \\
\hline Fatty acid $(\mu \mathrm{mol} / \mathrm{g})$ & & & \\
Total & $89.4 \pm 7.6$ & $69.1 \pm 10.0^{\mathrm{b}}$ & $66.8 \pm 6.9^{\mathrm{b}}$ \\
Acetic & $49.4 \pm 5.6$ & $36.5 \pm 7.6^{\mathrm{a}}$ & $34.7 \pm 4.9^{\mathrm{b}}$ \\
Propionic & $19.6 \pm 1.5$ & $15.2 \pm 2.2^{\mathrm{b}}$ & $15.0 \pm 1.8^{\mathrm{b}}$ \\
$n$-Butyric & $13.5 \pm 1.9$ & $10.7 \pm 2.0$ & $11.0 \pm 1.2^{\mathrm{a}}$ \\
$n$-Valeric & $2.2 \pm 0.2$ & $2.0 \pm 0.3$ & $1.9 \pm 0.3$ \\
iso-Butyric & $2.6 \pm 0.4$ & $2.6 \pm 0.4$ & $2.8 \pm 0.2$ \\
iso-Valeric & $2.0 \pm 0.4$ & $2.2 \pm 0.3$ & $1.9 \pm 0.2$ \\
$\mathrm{pH}$ & $7.4 \pm 0.4$ & $7.9 \pm 0.1^{\mathrm{b}}$ & $7.8 \pm 0.3$ \\
\hline
\end{tabular}

Data are mean $\pm \mathrm{SD}(n=5)$.

a, b Significant difference from 5\% corn oil group at $p=0.05$ and 0.01 , respectively; no difference between $5 \%$ butter group and beef tallow group.

groups. Although we have no information concerning the effect of dietary fats of standard contents on the intestinal microflora, Graber et al (4) showed that $C$. perfringens isolations were higher in rats given a diet with high $(40 \%)$ butter than a diet with high $(40 \%)$ corn oil. Moore et al (7) reported that the bacterial composition of the fecal microflora in pigs differed between high fiber and high corn oil diets.

The concentrations of acetic and propionic acids in the cecal contents were significantly lower in the rats fed beef tallow and butter diets $(p<0.05)$ than in the animals fed the corn oil diet, and the butyric acid concentration also showed a considerable, but not significant, decrease in the beef tallow $(p<0.05)$ and butter $(p=0.054)$ groups as compared to the corn oil group. Therefore, the total SCFA concentration of the cecal contents was significantly lower in the beef tallow and butter $(p<0.01)$ groups compared to the corn oil group. The concentrations of other minor SCFAs were not different among the experimental groups. The present data suggest that beef tallow and butter oil have suppressive effects on the production of SCFA by cecal bacteria in rats. The cecal $\mathrm{pH}$ values of the three animal groups tended to be inversely proportional to the total SCFA concentrations. Dietary corn oil may be more favorable for bacterial fermentation in the cecum of rats than dietary butter and beef tallow. This may be ascribed to the findings that the animals given corn oil had larger numbers of lactobacilli, bifidobacteria, and clostridia than did the animals given butter and beef tallow. As fat and fatty acids are known to inhibit bacterial growth in vitro $(3,5,10$, 13, 14), dietary fats are important factors to regulate the intestinal microflora, which is especially important in malabsorption of fats.

\section{REFERENCES}

(1) Carrol KK: Experimental studies on dietary fat and cancer in relation to epidemiological data. In Dietary Fat and Cancer, Ip C, Birt DF, Rogers AE and Mettlin C eds, Alan R. Liss, Inc, New York, 1986, p. 231-248

(2) Fay JP and Farias RN: The inhibitory action of fatty acids on the growth of Escherichia coli. J Gen Microbiol 91: 233-240, 1975

(3) Fuller R and Moore JH: The inhibition of the growth of Clostridium welchii by lipids isolated from the contents of small intestine of the pig. J Gen Microbiol 46: 23-41, 1967

(4) Graber CD, O'Neal RM and Rabin ER: Effect of high fat diets on intestinal microflora and serum cholesterol in rats. J Bacteriol 89: 47-51, 1965

(5) Kabara JJ, Swieczkowski DM, Comley AJ and Truant JP: Fatty acids and derivatives as antimicrobial agents. Antimicrob Agent Chemother 2: 23-28, 1972

(6) Mitsuoka T, Sega T, Yamamoto T and Yamamoto $\mathrm{S}$ : Eine vergleichende Methodik der qualitativen und quantitativen Analyse der Darmflora von Menschen und Tieren. Zentralbl Bakteriol Parasitenkd, Infektionskr Hyg, I Abt Orig A195: 455-469, 1965

(7) Moore WEC, Moore LVH, Cato EP, Wilkins TD and Kornegay ET: Effect of high-fiber and high-oil diets on the fecal flora of swine. Appl Environ 
Microbiol 53: 1638-1644, 1987

(8) Morishita Y and Miyaki K: Effects of age and starvation on gastrointestinal microflora and the heat resistance of fecal bacteria in rats. Microbiol Immunol 23: 455-470, 1979

(9) Morishita Y, Yamada H, Shiiba K, Kimura N and Taniguchi H: Effect of hydrolyzate of wheat bran hemicellulose on the cecal microflora and short-chain fatty acid concentrations in rats and mice. Bifidobacteria Microflora 12: 19-24, 1993

(10) Nieman C: Influence of trace amounts of fatty acids on the growth of microorganisms. Bacteriol Rev 18: 147-163, 1954
(11) Powell HJ and May JT: Effect of various lipids found in human milk on the growth of infant bifidobacteria. J Gen Appl Microbiol 27: 185-193, 1981

(12) Reddy BS: Amount and type of dietary fat and colon cancer: Animal model studies. In Dietary Fat and Cancer, Ip C, Birt DF, Rogers AE and Mettlin C eds, Alan R. Liss, Inc, New York, 1986, p. 295-309

(13) Smith HW: The antimicrobial activity of the stomach contents of suckling rabbits. J Pathol Bacteriol 90: 495-513, 1966

(14) Tsuchida M and Morishita Y: Antibacterial activity and bacterial degradation of linoleic and hydroperoxide. Bifidobacteria Microflora 14: 67-74, 1995 